\title{
Enhanced Uplink Scheduling for Continuous Connectivity in High Speed Packet Access Systems
}

\author{
Saied M. Abd El-Atty ${ }^{1}$, Konstantinos Lizos ${ }^{2}$ \\ ${ }^{1}$ Department of Computer Science and Information, College of Arts \& Science, Salman Bin Abdulaziz University, \\ Wadi Addawasir, KSA \\ ${ }^{2}$ Head of IT Department, Embassy of Greece Nobels Gate 45, Oslo, Norway \\ Email: sabdelatty@gmail.com, klizos@mfa.gr
}

Received September 21, 2011; revised June 15, 2012; accepted July 10, 2012

\begin{abstract}
The efficiency of high speed packet access (HSPA) systems is mainly based on channel quality information (CQI) reports transmitted by user equipment (UE) to Node B. In this paper, we propose an improved CQI prediction scheme based on a finite state Markov chain (FSMC) model for wireless channel, in order to reduce CQI signaling overhead in the HSPA system. Then, we introduce an enhanced uplink packet scheduling (EUPS) scheme to provide quality of services (QoS) guaranteed for continuous packet connectivity in the enhanced uplink (EUL). EUPS serves the active UEs not only according to buffer status but also according to reported state of the wireless channel of each UE. The performance of the proposed scheme in terms of average packet delay, average packet drop and average cell throughput is compared to the classical scheduler of 3GPP standards. The simulation results show the effectiveness of the proposed scheme.
\end{abstract}

Keywords: EUL; Scheduling; CQI; FSMC; HSPA

\section{Introduction}

Nowadays, HSPA is the best communication system to provide different services with QoS guaranteed to UEs at any time, any place, and supporting full mobility in a cost-effective and efficient manner. HSPA is also the best available radio-based technology when considering high download and upload speeds. The uplink is becoming increasingly important, with many UEs interested in uploading their clips onto online streaming providers and similar video-sharing sites [1]. The enhanced uplink (EUL) of WCDMA or commercially called high speed uplink packet access (HSUPA) is deployed in mobile marketing at the end of 2009. The main beneficial of the HSUPA is not only providing high uplink spectral efficiency compared to R99 UMTS systems but also delivering high data rate at the end users [2].

The scheduling procedure in HSPA systems is based on a set of rules that contains signalling control information in the downlink and uplink between Node B and UEs. The high speed downlink packet access (HSDPA) scheduling is mainly based CQI reports that are transmitted from UEs to Node B, in order to achieve high system efficiency. On the other hand, the HSUPA utilizes fast scheduling, hybrid automatic repeat request (HARQ), and shorter transmission time interval TTI (2 $\mathrm{ms}$ ) in order to enhance system throughput and minimize transmission delay [3]. However, according to 3GPP standards, the HSUPA scheduler at Node B is mainly based on the scheduling information (SI) that contains logical information, related to the power transmission and buffered data of UEs. As a result, the scheduling procedure for multimedia applications packet data does not quite guarantee the delay requirements of different services. Also in HSPA systems, CQI is periodically reported from UEs to Node B. However, the frequent CQI reports increase uplink interference, reducing the signal reception quality at the uplink, causing degradation of the system spectral efficiency. Hence, the uplink scheduler experiences some challenges to serve continuous packet connectivity and guarantee the QoS requirements of the multimedia traffic flows. Therefore, our goal in this work is to improve the performance efficiency of HSPA systems, hence we propose an improved CQI reporting scheme to minimize the signalling overhead and then increase the uplink reception quality. Subsequently, based on the proposed CQI reporting scheme, we adopt an EUPS scheme for packet data transmission on the EUL or HSUPA. The packet scheduling procedure in EUPS does not only take into account the delay sensitivity but also consider the fluctuating channel conditions. Therefore, the deployment of EUPS in HSPA systems would 
provide an opportunity for UEs in order to guarantee the QoS requirements i.e., lessen average packet delay as well as increase cell throughput.

The remainder of the paper is organized as follows. In Section 2 related work and problem statement are investigated. The proposed CQI reporting scheme and the prediction mechanism are presented in Sections 3 and 4 respectively. In sequel, the details of the EUPS are investigated in Section 5. The simulation results and performance evaluation are presented in Section 6. Finally, we conclude this work at Section 7.

\section{Related Work and Problem Statement}

Scheduling packet data transmission on the enhanced dedicated channel (E-DCH) in HSPA systems can be performed by time or code division scheduler [4]. In enhanced uplink (EUL) or namely HSUPA, code scheduling grants a fair radio resource with low data rate to multiple UEs continuously while time scheduling allocates radio resource to different UEs according to channel state information [5]. However, the noise rise level may be increased at Node B above the desired level in both types. A combined scheduler between code and time division scheduling is proposed in [4]. In addition, hybrid scheduling (HS) for resource allocation in HSUPA is presented in [6]. HS consists of traffic classifier, QoS monitor, queue selector and adaptive weight factor. Although, HS provides a QoS guaranteed service for mixed multimedia according to traffic characteristics and packet drop rate, the scheduler design and implementation is complex. On the other hand, the time scheduler in HS does not take into account the channel conditions of UEs.

In 3GPP standards [3], the classical scheduling (CS3gpp) operation at the E-DCH is based on the scheduling information (SI) and the happy bit (HB) during the transmission of the uplink logical channels when the UEs request the required resources from the Node B [2]. Accordingly, HSUPA scheduling procedure depends on downlink and uplink signalling data. In the downlink, a resource indication (scheduling grant) is required to notify the UE on the limited amount of uplink resources it is allowed to use. Scheduling grants (SG) carry different control signals such as maximum allowed E-DCH Dedicated Physical Data Channel (E-DPDCH)/Dedicated Physical Data Control Channel (DPCCH) power ratio of the active UEs and E-DCH TFC selection algorithm. The SG is performed once per TTI by using absolute grant and relative grant.

In every E-DCH transmission, UE transmits (SI) in the uplink logical channels containing the following information:

1) SNPL: denotes to the path losses of the serving cell and neighbour cells, the reserved size is 5 bits.
2) TEBS: denotes to the total buffer occupancy status of UE, the kept size is 5 bits.

3) UPH: denotes to the power available to the UE, the kept size is 5 bits.

4) HLID: denotes to the highest logical channel identification that is used to identify priority logical channel, the kept size is 5 bits.

5) HLBS: denotes to the highest priority logical buffer status, used for indicating the usage status of the highest logical priority channel buffer and the percentage of the total buffer being used, the kept size is 5 bits.

In addition to the above SI, the UE in each E-DPCCH transmission instance should send happy bit (HB) in order to indicate if UE asks more resources or not. The UE will indicate that is unhappy when the following states are met:

- UE is transmitting as much data as allowed by the current (SG); and

- UE has enough power available to transmit at higher data rate;

- Total buffer states would require more than Happy Bit-Delay_Condition ms to be transmitted with the Current_Serving_Grant $\times$ the ratio of active processes to the total number of processes.

In sequel, the Node B scheduler can control the power transmission and data rate to each UE. Therefore, in 3GPP standards the classical scheduler CS-3gpp at Node $\mathrm{B}$ is mainly based on the uplink and downlink signalling, carrying SI and HB information. However, when the numbers of UEs increase in the serving cell, the amount of the allocated resources per UE is decreased due to the increase in the uplink interference causing degradation of the cell throughput. On the other hand, the SI enables only the Node B scheduler to prioritize the traffic flows of an UE according to the information carried on logical channel and the buffered data, while it does not take into account the delay experienced by each UE. Therefore, the classical scheduler in 3GPP standards does not support the delay sensitivity of multimedia traffic $[2,3]$.

The main aim of this work is to improve the uplink reception quality and to reduce the signalling overhead by introducing a prediction CQI (P-CQI) reporting scheme that reveals the measured signal and interference noise ratio (SINR) of the active UE. Subsequently, based on the 3 GPP standards, we present an enhanced uplink packet scheduling (EUPS) in order to serve the traffic flows of UE, taking into account not only buffered status but also the quality of its channel. In addition, we consider the UE plays various applications with different QoS requirements and then the EUPS priorities those applications based on the experienced delayed data and their channel quality. Therefore, the service priority criterion in EUPS is able to serve different UE traffic flows corresponding to the delay sensitivity and the reported 
predicted channel state. In conclusion, EUPS guarantees continuous packet connectivity with different QoS requirements of the selected UE.

\section{New CQI Reporting Scheme}

In Release 5 [7], 3GPP proposed a periodic CQI feedback scheme as illustrated in Figure 1(a) that has a report cycle of $r_{c}$. The possible values of $r_{c}$ are [0,2, 10, 20, $40,80,160]$ msec. The Enhanced CQI reporting (E-CQI) scheme described at Release 6 extends Release 5 specifications by introducing additional CQI reports during periods of downlink activity. As shown at Figure 1(b) the additional CQI reports are transmitted with every packet acknowledgment (ACK) (and/or Non-ACK). The aim of the "Enhanced CQI Reporting" is to use longer report cycles compared to the periodic CQI scheme and increase the number of the CQI reports when it is needed (i.e. when the downlink activity increases).

Obviously, the shorter the report cycle is the more efficient performance of HSPA, as it provides better adaptive modulation coding (AMC) adaptation to the variations of the wireless channel. However, at the same time, the frequent CQI signalling increases uplink interference and thus decreases the average UE throughput as well as the achievable energy-per-bit to noise $E_{b} / I_{0}$ ratio at the enhanced uplink E-DCH. Therefore, we propose an improved CQI reporting scheme which aims to reduce the required CQI signaling even when the downlink data activity is relatively high, by employing a prediction CQI (P-CQI) scheme. According to this scheme, Node B predicts all the intermediary CQI reports between two subsequent CQI reports by utilizing a FSMC model of the wireless channel. Thus, as shown in Figure 1(c), in our proposed mechanism a number of CQI reports can be predicted instead of transmitted.

Adopting the simplified interference analysis presented in [8], we can measure the benefit of using P-CQI scheme. Considering $M_{u}=30$ the number of users in the cell, we calculate an approximate estimation of the uplink signal reception quality gain at the Node B. Figure 2 shows how the gain of the $E_{b} / I_{0}$ ratio increases as the ratio of predicted CQI reports to the total number of CQI reports, increases. The Periodic-CQI scheme with a reporting cycle of $2 \mathrm{~ms}$ is used as a reference base. As we can see in Figure 2, the reception quality of HSUPA improves as the number of CQI predictions increases. Although a high ratio of CQI predictions may be impractical in a real system, as prediction accuracy decreases

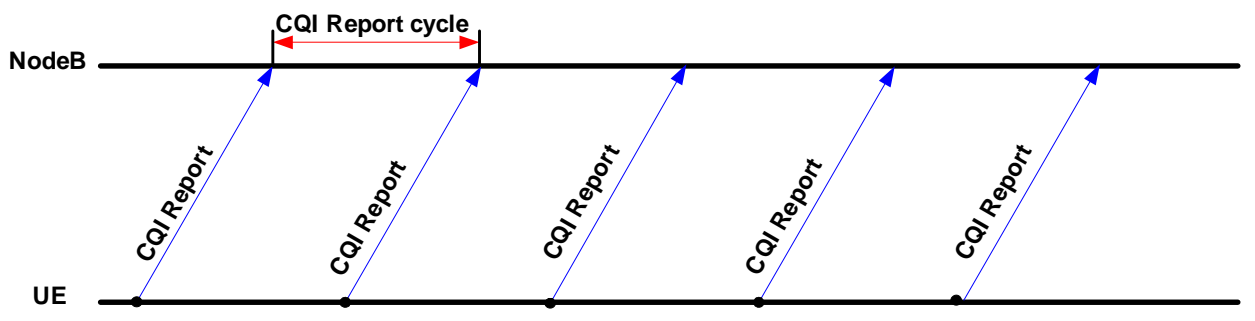

a) Periodic CQI reporting scheme

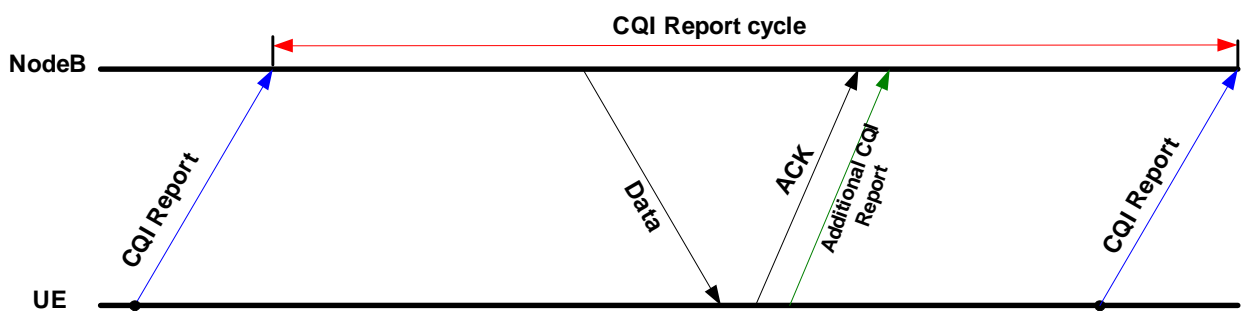

b) Enhanced CQI reporting scheme

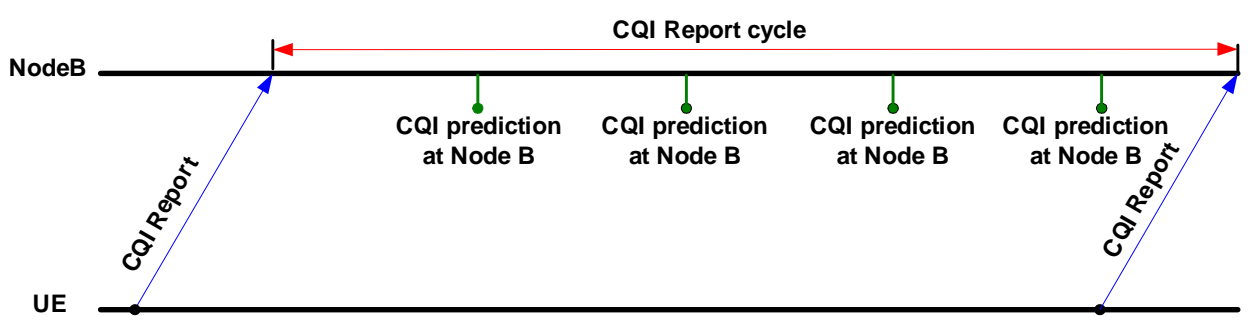

c) Prediction based CQI reporting scheme

Figure 1. CQI reporting schemes. 


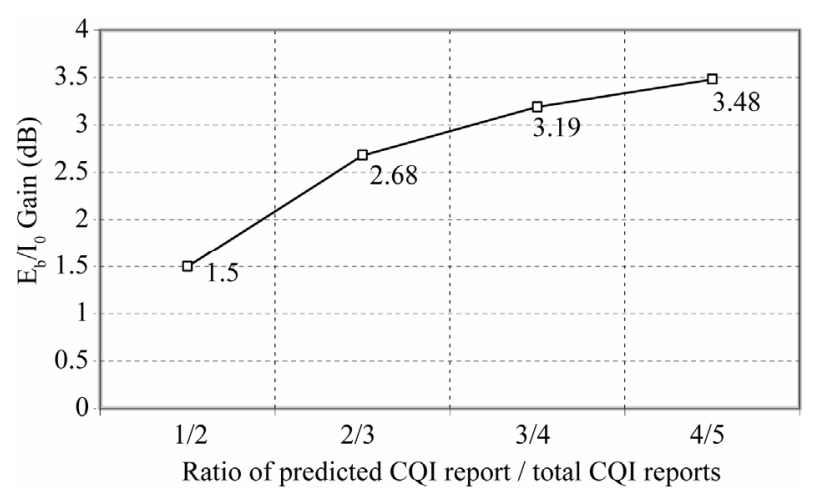

Figure 2. $E_{b} / I_{0}$ gain vs increasing ratio of CQI predictions in HSPA.

when the number of predicted samples is increased, we can conclude from Figure 2 that even by predicting, and thus avoiding, the transmission of just one every two CQI reports we can achieve a significant gain of approximately $1.5 \mathrm{~dB}$.

\section{Prediction Mechanism Based-CQI Scheme}

The CQI reports indicate the requested TFC and thus they reflect their current channel conditions. Therefore, they can be interpreted to SINR measurements. The obtained SINR values are then used by the P-CQI scheme to predict, through a FSMC model, the next state of the wireless channel and therefore the next CQI. Consequently, predicting the next CQI at the Node B helps in reducing the number of the required CQI reports.

\section{Modeling Wireless Channel Based-FSMC}

We consider a multipath fading environment, in which the received $\operatorname{SINR}\left(\gamma_{i}\right)$ of an active $\mathrm{UE}_{i}$ is proportional to the square of the signal envelop. The probability density function (pdf) of $\gamma_{i}$ can be expressed as in [9].

$$
p\left(\gamma_{i}\right)=\frac{1}{\gamma_{0}} e^{-\gamma_{i} / \gamma_{0}}, \gamma_{i}^{3} \geq 0
$$

where $\gamma_{0}=E\left\{\gamma_{i}\right\}$ is the mean value of SINR, which can estimated by averaging the SINR of all $M_{u}$ active UEs in the E-DCH cell as follows:

$$
\gamma_{0}=\frac{\sum_{j \in M_{u}} \gamma_{j}}{M_{u}}
$$

According to the FSMC model depicted in Figure 3, $\gamma$ can be partitioned into $K$ non-overlapping intervals by thresholds $\Gamma_{k}, k \in\{0,1,2, \cdots, K\}$, the wireless channel of active $\mathrm{UE}_{i}$ is considered to be in state $S_{k}$ if the measured $\gamma_{i}$ lies in the interval $\left\{\Gamma_{k}, \Gamma_{k+1}\right\}$. Assuming that the channel fades slowly with respect to CQI feedback report cycle $\left(r_{c}\right)$ and Doppler shift $f_{d}$ in the carrier frequency $f_{c}$,

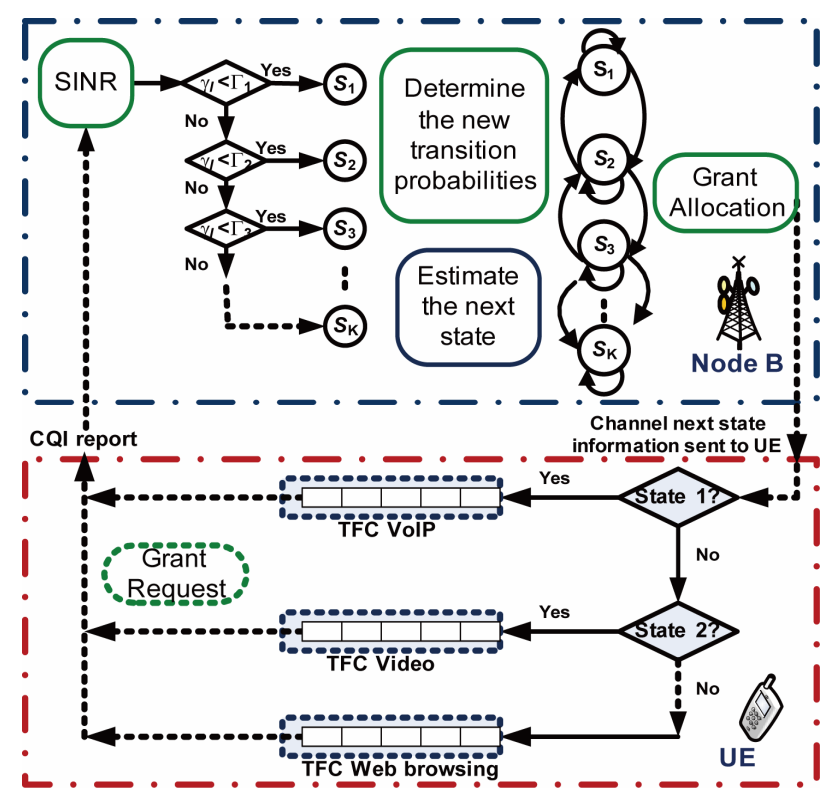

Figure 3. P-CQI scheme in HSPA system.

is $f_{d}=v f_{c} / c$, then probability of transmission in state $k$, can be obtained as follows [9]:

$$
\pi_{k}=\int_{\Gamma_{K}}^{\Gamma_{K+1}} p(\gamma) \mathrm{d} \gamma=e^{-\frac{\Gamma_{k}}{\gamma_{0}}}-e^{-\frac{\Gamma_{k+1}}{\gamma_{0}}}
$$

Taking into account the previous equations as well as the fundamental theory of Markov chains and since the summation of all transition probabilities equals 1 , it is possible to determine the steady state transition probabilities from state $k$ to $k+1$ or $k-1$ or remaining in state $k$. Hence, the derived transition probability of transmissions at the FSMC model can be approximated by the following equations:

$$
\begin{aligned}
& p_{k, k+1} \cong \frac{1}{\pi_{k}} f_{d} T_{s} \sqrt{\frac{2 \pi \Gamma_{k+1}}{\gamma_{0}}} e^{-\frac{\Gamma_{k+1}}{\gamma_{0}}} \\
& p_{k, k-1} \cong \frac{1}{\pi_{k}} f_{d} T_{s} \sqrt{\frac{2 \pi \Gamma_{k}}{\gamma_{0}}} e^{-\frac{\Gamma_{k}}{\gamma_{0}}} \\
& p_{k, k}=1-\left(p_{k, k+1}+p_{k, k-1}\right)
\end{aligned}
$$

Therefore, the predicted state for each UE can be transmitted via the high-speed dedicated physical control channel (HS-DPCCH) in the uplink every TTI $(2 \mathrm{~ms})$. Since $k$ is the current state of the channel, computed by a SINR report, the next transition state, namely $m$, according to the highest state transition probability of a Markov chain model is as follows:

$$
p_{k, m}=\max \left(p_{k, k}, p_{k, k+1}, p_{k, k-1}\right)
$$

More future states may be predicted in the same manner using the same calculated transition probabilities. The 
state transition probabilities are updated periodically based on the real SINR levels received from the UEs at each time the real CQI measurements are collected. By this method the UE is able to select the appropriate application for transmission based on the reported state. For instance, as shown in Figure 3, when Node B reports the predicted state $\left(S_{1}\right)$ to UE, the UE selects the TFC of the VoIP application and so on.

\section{Enhanced Uplink Packet Scheduling (EUPS) Scheme}

The aim of proposed scheme is to reduce the CQI signaling overhead and thus improve the signal reception quality at the enhanced uplink (EUL). Therefore, the principle of EUPS procedure on E-DCH is governed by the proposed P-CQI reporting mechanism and at the same time the operation of EUPS is still certified on 3GPP standards.

We consider that the UE is able to play different applications of multimedia traffic while UE can be notified to send SINR measurement reports periodically to Node $\mathrm{B}$ during the roaming in the serving cell. According to the measured SINR, the EUPS predicts the next state channel by using the P-CQI scheme as shown in Figure 3. The UE sends the required data rate of each application to Node B through grant request (GR). Depending on the received GR and the predicted states of UE channels, the EUPS examines the available resources, computes the Transport Format Combination Set (TFCS) and signals the TFCS to the UE via grant allocation (GA). TFCS is the set of Transport Format Combinations (TFCs) that can be used by the UE for data transmission.

On the other hand, EUPS employs a priority criterion $(P C)$ at Equation (8) based on the delay sensitivity and the channel quality for data transmission of each flow in the E-DCH. $P C$ is defined as the ratio of Head of Line packet delay over the packet delay threshold of a flow. In addition, we consider the successful probability of flow transmission as a discriminated priority between the delayed flows. Therefore, the service of the highest priority logical channel ID (HPLC) of flow transmission during each TTI ( $2 \mathrm{~ms})$ can be expressed as follows:

$$
P C\left(H P L C_{i}\right)=\left(\frac{H O L_{i}}{D_{t h, i}}\right) \cap P_{s, i} \quad i \in 1,2,3
$$

where $H O L_{i}$ denotes the Head of Line packet delay of flow $i$ and $D_{t h, i}$ is the delay threshold of packets for flow $i \in 1,2,3$ that referred to VoIP, Video, and Web browsing respectively. $P_{s, i}$ is the successful probability of bit transmission during the next sub-frame and is determined through prediction mechanism of the wireless channel. The scheduling procedure of EUPS is performed every TTI corresponding to the service priority. The EUPS steps as follows:

1) Set a list of UEs prioritization according to $P C$, solve ties based on $P_{s}$.

2) Select the highest priority UE from the list and assign a Scheduling Grant (SG) and,

3) Exclude any UE, not being able to get SG from the current TTI, due to power limitations.

4) For all the remaining of UEs in the prioritization list, share the residual capacity according to the following steps:

- Set UEs in decreasing priority list according to TEBS. Solve ties based on $P_{s}$.

- Assign a SG according to the highest power transmission.

- End the assignment of SG when the residual capacity is not sufficient.

Finally, data transmission takes place on the uplink E-DCH Dedicated Physical Data Channel (uplink EDPDCH). Subject to the amount of data in the buffer and on the required transmission power, the UE selects the most appropriate TFC between those included in the TFCS. In addition, EUPS makes use of computing $P_{s, i}$ of each flow, to avoid erroneous packet transmissions and thus increases the bandwidth utilization. Thus computing $P_{s, i}$ is preferable instead of employing HARQ and power headroom requiring higher power requirements, especially when channel conditions have worsened. Therefore, EUPS guarantees continuous packet connectivity to UE corresponding to the predicted state reported via HSDPCCH.

\section{Simulation and Performance Evaluation}

The performance evaluation of the proposed scheme based on the P-CQI prediction mechanism is driven by developing a system level simulator for enhanced uplink in $3 \mathrm{GPP}$. We compare the performance of EUPS with a conventional scheduler based on 3GPP standards (CS3gpp) [3] and the hybrid scheduling (HS) algorithm [6]. We evaluate the performance of EUPS in terms of average packet delay, average packet drop rate and cell throughput.

The simulation scenario was saturated with realistic parameters to reflect forecasted growth of UE future traffic requirements. We consider a cell with a radius of 1 $\mathrm{km}$. Node B is located at the centre of the cell. Each UE is uniformly distributed in the cell and can play mixed traffics. A mixed multimedia traffic includes VoIP, Video and Web browsing [10] with average data rates $20 \mathrm{kpbs}$, $60 \mathrm{kpbs}$, and $90 \mathrm{kbps}$ respectively. The proportions of the multimedia traffics for VoIP, Video and Web browsing are $70 \%, 20 \%$ and $10 \%$ respectively.

As we mentioned previously, the operation of EUPS is based on the proposed P-CQI scheme corresponding to 
the instantaneous value of SINR of UEs, then the interference generated at neighbouring cells can be reduced, and cell throughput is increased. Furthermore, for the evaluation the average packet drop rate, we consider the average packet error probability $(P E R)$ when the application $i$ is transmitting, depends solely on the bit errors and the data rate. It can be expressed as follows:

$$
P E R_{i}=1-\left(P_{s, i}\right)^{R_{i}}
$$

where $R_{i}$ is the transmission rate of flow and $P_{s, i}$ is computed as follows:

$$
P_{s, i}=1-\frac{1}{\pi_{k}} \int_{\Gamma_{k}}^{\Gamma_{k+1}} p_{e m}\left(\gamma_{i}\right) \cdot p\left(\gamma_{i}\right) \mathrm{d} \gamma_{i}
$$

where $p_{e m}(\gamma)$ denotes the error probability for a specific modulation scheme in AMC map. By computing the PER at each TTI, EUPS can determine the average packet drop rate $\left(P_{d, i}\right)$ of traffic flow $i$ as follows:

$$
P_{d, i}=\frac{(\text { No. of transmitted packets })_{i} \times\left(1-P E R_{i}\right)}{(\text { total No. of transmitted pacekts })_{i}}
$$

Simulations have initially been run for an average number of UEs per cell equal to 50 and for a pedestrian profile at $3 \mathrm{~km} / \mathrm{h}$ [11]. The session arrival rate is modelled by a Poisson process. The traffic load increases by increasing the number of UEs in the serving cell. The initial location of each UE is randomly distributed in the cell; the direction of UE movement is uniformly distributed. The macrocell propagation model is adopted for calculating the path loss at distance $d_{i}(\mathrm{~km})$ from Node B. Therefore, the attenuation $L_{p}$ of the transmitted signal for a Node B antenna height of 15 meter and $2.1 \mathrm{GHz}$ carrier frequency is defined as $L_{p}\left(d_{i}\right)=128.1+37.6 \log _{10}\left(d_{i}\right)[\mathrm{dB}]$.

The modelling of the wireless channel of UE is performed through a four-state FSMC which provides the required accuracy without adding excessive complexity. We employed equal probability method (EPM) [9] for estimating the steady state probabilities and afterwards the transition probabilities. The CQI signalling delay is not taken into account in the process of evaluating packet delay and it is still open issue for studying in HSPA systems.

At the uplink, the UE's data are transmitted through E-DCH Dedicated Physical Data Channel (uplink EDPDCH) with the selected TFC while the HS-DPCCH is employed for fast power control and signaling data. The detailed simulation parameters are summarized in Table 1. We now present a snapshot of our simulation results, which provide fairly interesting insight and performance efficiency compared to the previous works $[3,6]$.

\subsection{Average Packet Delay Comparison}

Figures 4 and $\mathbf{5}$ show the average packet delay of VoIP
Table 1. Basic simulation parameters in HSPA systems.

\begin{tabular}{cc}
\hline Parameter & Value \\
\hline Carrier Freq. & $2.1 \mathrm{GHz}$ \\
Ch. Bandwidth & $5 \mathrm{MHz}$ \\
TTI & $2 \mathrm{~ms}$ \\
Cell Radius & Hexagonal, $1 \mathrm{~km}$ \\
UE distribution & Uniform \\
Channel Models & Rayleigh \\
Traffic Models & VoIP, Video, Web browsing \\
Path Loss Model & $L_{p}\left(d_{i}\right)=128.1+37.6$ log ${ }_{10}\left(d_{i}\right)[\mathrm{dB}]$. \\
Thermal Noise & $-174 \mathrm{dBm} / \mathrm{Hz}$ \\
UE Tx power & $20 \mathrm{dBm}(0.1 \mathrm{Watt})$ \\
Power allocation Tx power & Control $20 \%$ E-DPDCH $80 \%$ \\
Other-to-Own cell & from total power \\
interference ratio & 0.5 \\
Iterations per simulation run & $7600 \mathrm{sec}$ \\
Simulation time &
\end{tabular}

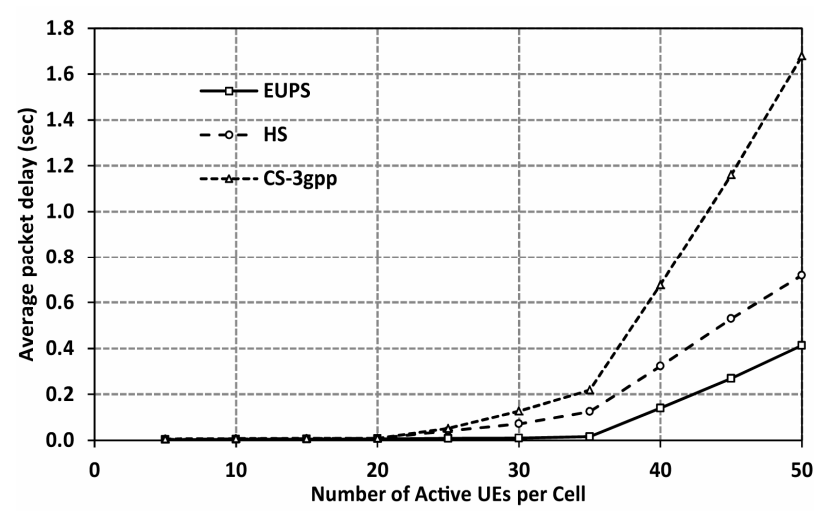

Figure 4. Average packet delay for VoIP traffic.

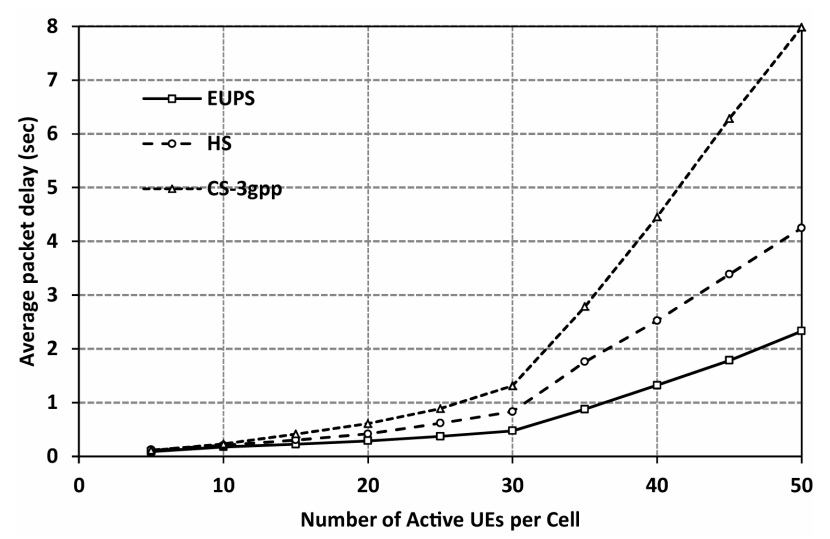

Figure 5. Average packet delay for Web browsing traffic. 
and Web browsing traffic flow respectively with increasing the number of UEs per cell. As expected, by increasing the number of active UEs in the cell, the average packet delay is increased for both VoIP and Web browsing traffic services. However, by employing EUPS a significant delay reduction in both cases is achieved. This occurs on the basis of the priority criterion $(P C)$ where it can sort the highest priority logical channels not only according to the delay sensitivity but also according to the reported predicted channel state of each UE.

In addition, if two UEs have the same delay, EUPS serves the UE with the higher successful transmission probability $\left(P_{s}\right)$. Therefore, the number of UEs awaiting service is decreased. This leads to a reduction of the delayed packet in the UEs buffers'. For comparison reasons, assuming an average number of UEs $=25$ in the cell as shown at Figure 4 of VoIP traffic, the average packet delay is reduced by approximately $11 \%$ compared to HS and CS-3gpp schemes.

\subsection{Average Packet Drop Rate Comparison}

Figures 6 and 7 show the average packet drop rate, for VoIP and Web browsing traffic services respectively with increasing the number of active UEs per cell. As illustrated in the Figures 6 and 7 the EUPS scheme is capable of reducing the packet drop rate of the scheduled traffic flow for VoIP or Web browsing compared with CS-3GPP and HS disciplines.

Also besides the reduction of delayed packets, EUPS exploits the computing of $P_{s}$ in order to minimize the retransmission of erroneous packet and hence the average packet drop rate is significantly decreased. Subsequently, the bandwidth utilization is increased and the cell throughput is increased compared to CS-3GPP and HS disciplines as shown in Figure 8.

To summarize the performance of the proposed scheme, "EUPS" is able to sort the traffic flows not only based on the delay sensitivity but also takes into account the reported channel states of UEs. Therefore, the packet delay and packet drop rate are significantly decreased. As well as when UE plays different multimedia traffic, EUPS can provide QoS guaranteed for continuous packet connectivity according to reported channel state.

\section{Conclusion}

In this paper, we have proposed an enhanced uplink packet scheduling (EUPS) scheme for multimedia applications packet data transmission in HSPA systems. EUPS operation based on an improved prediction CQI reporting scheme. The simulation results show that the performance efficiency of HSPA systems employed EUPS is superior compared to the conventional scheduler in $3 \mathrm{GPP}$ standards.

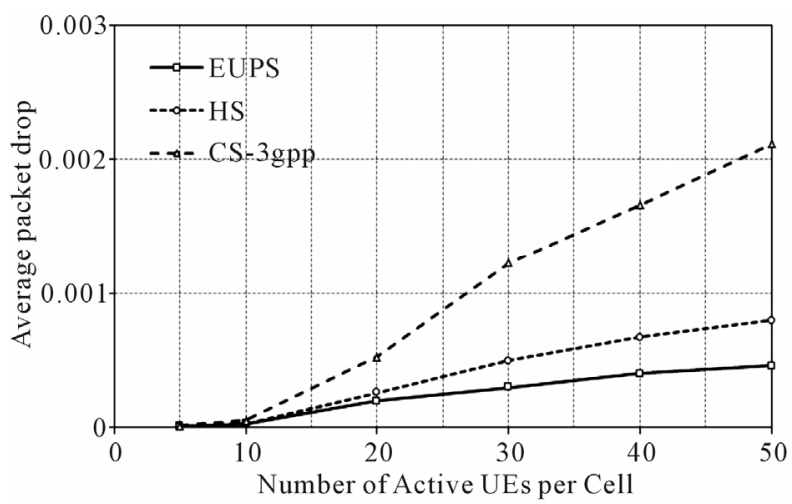

Figure 6. Average packet drop rate for VoIP.

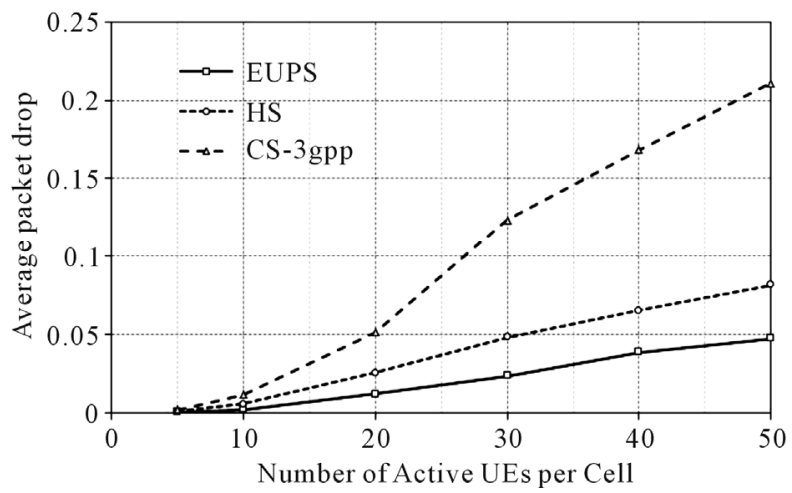

Figure 7. Average packet drop rate for web browsing traffic.

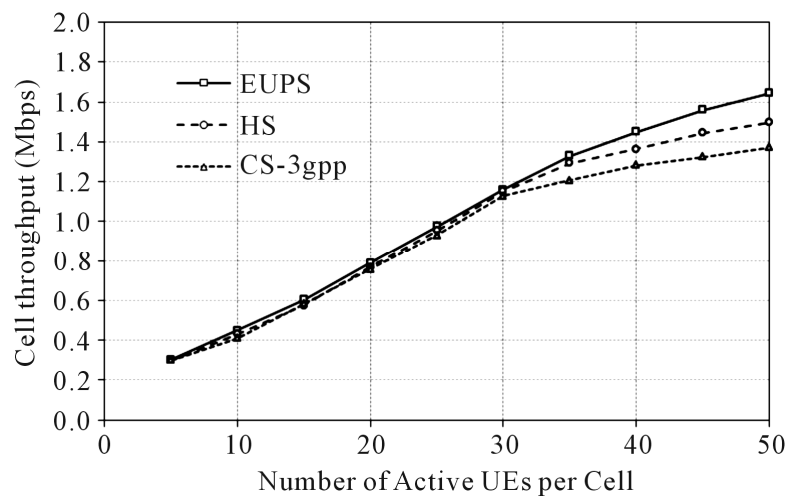

Figure 8. Comparison of cell throughput.

\section{Acknowledgements}

A part of this work is carried out in Communication LAB at Faculty of Electronic Engineering, Menoufia University, Egypt in the last summer.

\section{REFERENCES}

[1] "HSPA Brings the World," 2012. http://www.ericsson.com

[2] H. Holma and A. Toskala, "HSDPA/HSUPA for UMTS 
High Speed Radio Access for Mobile Communications," John Wiley \& Sons Ltd., Hoboken, 2006.

doi: $10.1002 / 0470032634$

[3] 3GPP TS 25.319 V7.2.0, "Enhanced Uplink; Overall Description; Stage 2 (Release 7)," 2007.

[4] C. Rosa, J. O. Carnero, T. B. Sørensen, J. Wigard and P. E. Mogensen, "Combined Time and Code Division Scheduling for Enhanced Uplink Packet Access in WCDMA," IEEE Proceedings of the 60th Vheicular Technology Conference, Los Angeles, 26-29 September 2004, pp. 851-855.

[5] D. C. Dimitrova, J. L. van den Berg, G. J. Heijenk and R. Litjens, "Flow Level Performance Comparison of Packet Scheduling Schemes for UMTS EUL," Proceedings of 6th International Conference on Wired/Wireless Internet Communications, Tampere, 28-30 May 2008, pp. 27-40.

[6] Y. J. Kang, J. Kim, D. K. Sung and S. Lee, "Hybrid Scheduling Algorithm for Guaranteeing QoS of RealTime Traffic in High Speed Uplink Packet Access
(HSUPA)," IEEE 18th International Symposium on Personal, Indoor and Mobile Radio Communications, Athens, 3-7 September 2007, pp. 1-5.

[7] 3GPP TR 25.858 V5.0.0, "High Speed Downlink Packet Access: Physical Layer Aspects (Release 5)," 2002.

[8] N. Fukui, "Study of Channel Quality Feedback in UMTS HSDPA," 14th IEEE Proceedings on Personal, Indoor and Mobile Radio Communications, 7-10 September 2003, pp. 336-340.

[9] Q. Q. Zhang and S. A. Kassam, "Finite-State Markov Model for Rayleigh Fading Channels," IEEE Transactions on Communication, Vol. 47, No. 11, 1999, pp. 1688-1692. doi:10.1109/26.803503

[10] 3GPP TR 25.896 V6.0.0, "Feasibility Study for Enhanced Uplink for UTRA FDD," 2004.

[11] ETSI, "Universal Mobile Telecommunications System (UMTS); Selection Procedures for the Choice of Radio Transmission Technologies of the UMTS," TR 101112 , V3.2.0, 1998. 